\title{
CALCULUS INFINITESIMALIS: UMA TEORIA ENTRE A RAZÃO E O MITO?
}

\section{Calculus infinitesimalis: a theory between reason and myth?}

\author{
Tadeu Fernandes de Carvalho ${ }^{1}$. Itala Maria Loffredo D'Ottaviano ${ }^{2}$
}

Resumo: Neste artigo percorremos parte dos caminhos históricos e lógico-matemáticos que unem o cálculo infinitesimal à análise não-standard de Abraham Robinson, e esta ao cálculo diferencial paraconsistente - um cálculo proposto por Newton da Costa, que tem como lógica e teoria de conjuntos subjacentes a lógica paraconsistente e a teoria paraconsistente de conjuntos. Exibimos, para esse cálculo, algumas propriedades e conceitos inéditos, incluindo um teorema de transferência que, de forma simplificada, estabelece as condições sob as quais um teorema do cálculo clássico é, também, um teorema do cálculo paraconsistente. Concluiímos com algumas considerações acerca do ensino do cálculo com ênfase na abordagem infinitesimal.

Palavras-chave: Cálculo infinitesimal. Cálculo paraconsistente. Análise não-standard. História. Costa, Newton da.

Abstract: In this paper we go through the historical, logical and mathematical paths which join infinitesimal calculus to the non-standard analysis of Abraham Robinson, and this to paraconsistent differential calculus - a calculus proposed by Newton da Costa, supported by paraconsistent logic and paraconsistent set theory. We present for this calculus, some unpublished concepts and properties, including a transfer theorem establishing the conditions under which a given formula is a theorem of classical calculus if, and only if, there is an "interpretation" of the same which is a theorem of paraconsistent calculus. We conclude the paper with some considerations about the teaching of Calculus with emphasis on use of infinitesimal approach.

Keywords. Calculus infinitesimalis. Paraconsistent calculus. Non-standard analysis. History. Costa, Newton da.

\footnotetext{
${ }^{1}$ Centro de Ciências Exatas, Ambientais e de Tecnologias (CEATEC), Faculdade de Matemática, Pontifícia Universidade Católica de Campinas (Puccamp). Rodovia D. Pedro I, Km. 136, Parque das Universidades. Campinas, SP, Brasil. 13.086-900. tadeu_fc@puc-campinas.edu.br

${ }^{2}$ Centro de Lógica, Instituto de Filosofia e Ciências Humanas, Universidade Estadual de Campinas. Campinas, SP, Brasil.
} 


\section{Introdução}

Uma das conhecidas cosmogonias da mitologia grega considera que os primeiros seres foram a Terra, Érebo, que personificava a escuridão primordial, e o Amor (Eros), nascido do ovo da Noite, no isolamento do Caos. Inspiradora, como tantas outras emanações da era mítica, ressoou entre os filósofos naturalistas da Escola Jônica, que floresceu em Mileto, na Ásia Menor, do século VI a.C até a sua destruição pelos persas, em 494 a.C. Foi assim que Tales de Mileto (gr. $\Theta \alpha \lambda \hat{\eta} \varsigma)$, (624-548 a.C.), seu fundador, elegeu a água como o elemento essencial, origem de todas as coisas. Reflexos de seu papel de introdutor do método científico podem ser encontrados em seus trabalhos na Geometria e na Astronomia. Anaximandro de Mileto (gr.

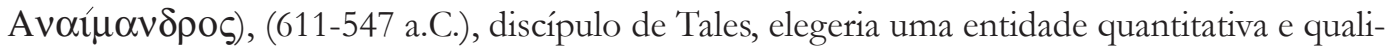
tativamente infinita, o a-peiron - algo sem princípio e nem fim, apenas perceptível pela mente.

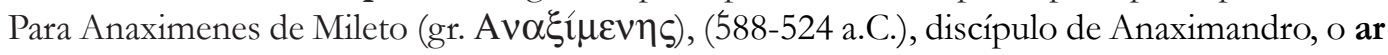
seria o elemento primordial, do qual todas as coisas derivariam por ação de sua condensação e rarefação, incluindo a própria alma. Sem a intervenção de Eros, a liberdade, a dúvida e a contestação, importantes propulsores do conhecimento, somadas a um misto embrionário de indução e dedução, e a resquícios do que Freud e Jung poderiam classificar de arquétipos da era mítica, criaram as condições necessárias para a arrancada da ciência grega. Mais alguns poucos séculos e a lógica e a física se integrariam ao conhecimento matemático grego que, à época, também flutuava no Caos. Provavelmente sob a influência de Tales, Pitágoras, por volta de 532 a.C, fundaria em Crotona, no sul da Itália, a Escola Pitagórica, relacionando fortemente a filosofia à política e à religião, e adotando o número como elemento primordial. Em sua numerologia, o 1 representava o elemento criador, em seu aspecto material, o 2 se associava à criação intelectual, e o 5, refletindo os antigos mitos, associava-se ao Caos. Sua classificação dos 4 saberes - aritmética, astronomia, geometria e música - produziria o quadrivium ou, do latim, quatro caminhos que, com o trivium ou três caminhos, compreendendo a lógica, a gramática e a retórica, comporia as sete artes liberais das universidades medievais. $\mathbf{O}$ trivium, como elenco de disciplinas, igualmente nos remete à Grécia clássica, particularmente aos trabalhos de Empédocles de Agrigento (492-432 a.C) na retórica, de Aristóteles (384-322 a.C.) na lógica, e dos filósofos alexandrinos na gramática.

Original de Estagira, colônia grega da Trácia, Aristóteles foi quem promoveu os maiores avanços científicos desde Tales, com a introdução do rigor sobre o método, criando a lógica e as bases da física e do método axiomático. Descrevendo e interpretando seus predecessores pitagóricos, Aristóteles destacaria o papel da mônada em sua filosofia, na qual estavam fortemente entrelaçadas a matemática e a religião. A mônada, sob o ponto de vista dos pitagóricos e de várias outras correntes filosóficas, é algo não apenas dotado de potencialidades criadoras mas, também, que possui percepção e vontade. A depender da variação dessas características, poderiam existir diferentes mônadas. Expressivo elemento justificado pela vontade e necessidade de compreender o mundo, ganhou interpretações variadas ao longo da história, mantendo, porém, de algum modo, o caráter unificador e de unidade. Na matemática dos pitagóricos, como na aritmética $(\alpha \rho \imath \beta \mu \eta \tau \imath \chi \alpha)$ e na logística

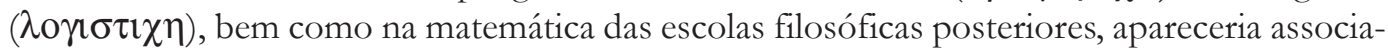
da com a figura do infinitésimo e com o Método da Exaustão, introduzido por Eudoxo de Cnido (390-338 a.C.). Esse método desempenhou, por longo tempo - como ilustram os traba- 
lhos de Apolonio de Perga (262-190 a.C.) referentes às cônicas e os trabalhos de Arquimedes, de quem falamos um pouco a seguir - papel que lembra aquele desempenhado pelo Cálculo Diferencial e Integral, na era moderna, em cálculos de áreas e volumes.

Na obra $O$ método (EVES, 2004), Arquimedes de Siracusa (287-212 a.C.) trava contato com Eratóstenes (285-194 a.C.), a quem encaminha problemas em busca de colaboração ou sugestões para as respostas. Eis como se inicia esse contato, no prefácio do Método, incluindo, na sequência, o primeiro problema:

Enviei-lhe em ocasião passada, alguns teoremas que descobri, restritos aos seus enunciados, convidando-o a descobrir as provas que até o momento não apresentei. Os enunciados dos teoremas que enviei são os seguintes:

Se em um prisma reto, tendo como base um paralelogramo, se inscreve um cilindro tendo as bases nos paralelogramos opostos, e os lados (i.e., quatro geradores) nos planos restantes [faces] do prisma, e se através do centro do círculo, que é base do cilindro e [através de] um lado do quadrado no plano oposto ao mesmo, um plano é traçado, o plano assim traçado irá produzir no cilindro um segmento que é limitado por dois planos e a superfície do cilindro, um desses dois planos sendo aquele que foi traçado e o outro, o plano em que a base do cilindro se encontra, e a superfície sendo aquela que se encontra entre os referidos planos; e o segmento "cortado" do cilindro é a sexta parte de todo o prisma.

Todas essas proposições já foram provadas. Além delas, usarei também a seguinte proposição, facilmente demonstrável:

Se em duas séries de magnitudes, as da primeira série são, na ordem, proporcionais às da segunda e, ainda, as magnitudes [da primeira série], todas ou algumas delas, estão em uma razão qualquer [para as magnitudes de uma terceira série], e se as magnitudes da segunda estão na mesma razão para as correspondentes magnitudes [de uma quarta série], então as somas das magnitudes da primeira estarão para a soma das magnitudes da terceira, na mesma razão que as somas das magnitudes da segunda estarão para a soma das magnitudes da quarta. (HEATH, 1912, p. 14-15, tradução nossa)

As provas mecânicas de Arquimedes, como pode ser visto nessa obra facilmente encontrável em livros e artigos, guardam, pelo uso do Método da Exaustão, grande afinidade com os cálculos de áreas e volumes desenvolvidos no Cálculo clássico. No entanto, analisando-se a prova da Quadratura da parábola, que aqui omitimos, verifica-se que isso não é tão natural como parece. Tanto Euclides quanto Arquimedes, no uso desse método, ajustam-no às características do pensamento grego, que incluem a predominância do finito sobre o infinito e da geometria e da estática sobre a análise e a dinâmica, respectivamente.

Avancemos mais na história, deixando para trás os mitos e verdades da Idade Média e o final do Império Romano, que, em sua derrocada, levou consigo alguns tesouros do conhe- 
cimento grego, e aportemos na própria Itália, na época de Galileu Galilei (1564-1642). Da mesma forma como se comunicaram Arquimedes e Eratóstenes, Johannes Kepler (1571-1630) e Galileu mantiveram contato para discutir e validar sua opiniões e trabalhos. Trabalhos que transformariam o conhecimento da física e da matemática, em uma época em que ousar era, em razão da intolerância religiosa, dar mostras de extrema coragem. Como bem se sabe, Galileu sofreu a censura da Igreja católica e a mãe de Kepler, Katharina, quase foi levada à fogueira, acusada de bruxaria. Giordano Bruno (1548-1600) teve, como se sabe, pior destino. Mas para nós interessa aqui, de fato, registrar que os infinitésimos estiveram na órbita de seus trabalhos físicos e matemáticos, direta ou indiretamente, como pode ser visto em Baron (2004).

Reencontraremos, um pouco à frente, as mônadas de Leibniz e os infinitésimos de Newton, mais uma vez sob motivação dos fenômenos naturais e dos desafios lógicos, matemáticos e filosóficos, cujas discussões movimentavam as universidades mais tradicionais da Europa, como a Universidade de Cambridge. A física de Newton dependeria essencialmente do Cálculo Infinitesimal, com o qual participaria da consolidação da nova ciência, moldada por Galileu.

Iremos aprofundar um pouco mais essas considerações predominantemente históricas, que cumprem aqui o papel de nos ajudar a situar convenientemente a evolução da matemática propiciada pelo emprego do infinitésimo como alternativa à teoria de limites. Começaremos por uma breve retrospectiva da evolução do cálculo infinitesimal, em síntese, uma teoria contida nessa nova matemática, passando pelo advento da análise não-standard (ANS) de Robinson (1961), até chegarmos ao cálculo diferencial paraconsistente de Newton da Costa (2000). Um cálculo que estende o cálculo clássico, para o qual introduzimos, em nossa tese de doutorado em filosofia (CARVALHO, 2004), uma série de resultados novos, alguns dos quais apresentaremos aqui. Não nos aprofundaremos em justificativas e demonstrações, mas, ainda assim, exibiremos, em sua linguagem, uma aplicação clássica à física: a aceleração no movimento circular uniforme.

A figura central na história do cálculo infinitesimal, reforçando o que já mencionamos anteriormente e como sugere a própria denominação, é o infinitésimo, que remonta à matemática e à filosofia gregas e está presente, por exemplo, nos paradoxos de Zenão de Elea (495-430 a.C.) e nos trabalhos de Eudoxo e de Arquimedes. Mas o próprio Eudoxo, não obstante interessado nos cálculos de áreas e de volumes, preferiu o emprego da matemática euclidiana, encerrando um longo período de aceitação, desde seu uso por Demócrito de Abdera (460-370 a.C.). Era o início de uma longa e acidentada jornada pela história da matemática.

Newton e Leibniz, historicamente apresentados como protagonistas da criação do Cálculo, envolveram, sob concepções distintas, os infinitésimos em seus trabalhos: Newton visando mais a física e os fenômenos naturais e Leibniz a lógica e a metafísica. Ambos encontraram problemas com aspectos lógicos, matemáticos e epistemológicos desses objetos. Do final do século XVII ao final do século XIX, período em que o uso dos infinitésimos esteve essencialmente restrito a aplicações informais na física, os mais destacados matemáticos europeus, principalmente, estiveram envolvidos no trabalho de desenvolvimento do cálculo diferencial e integral e da análise matemática. Destacam-se, aí, Bernhard Placidus Johann Nepomuk Bolzano (1781-1848), Augustin-Louis Cauchy (1789-1857) e Karl Theodor Wilhelm Weierstrass (1815-1897), diretamente responsáveis pelo desenvolvimento da teoria de limites. A crença de Georg Cantor (1845-1918) de que seria possível, através da Teoria de Conjuntos, 
ser provada a inexistência dos infinitésimos, foi um importante elemento a afastá-los do interesse de seus pares, por décadas. Seriam fundamentais, para alterar esse estado de desconfiança reforçado por Cantor, alguns trabalhos de Thoralf Albert Skolem (1887-1963) e de Jerzy Yos' (1920-1998).

Skolem, entre 1933 e 1934, introduziu um modelo não arquimediano para a aritmética, envolvendo números infinitos. Algo realmente desafiador, visto que, para a aritmética usual, vale a propriedade arquimediana, pela qual, para quaisquer números positivos $\mathrm{r}$ e $\mathrm{k}$, existe um número natural $\mathrm{n}$ tal que $\mathrm{k}+\mathrm{k}+\ldots+\mathrm{k}>\mathrm{r}$, $\mathrm{k}$ somado $\mathrm{n}$ vezes. Dito de outra forma, não importa quantas vezes $\mathrm{k}$ tenha de ser somado, assim procedendo o valor de $\mathrm{r}$ será ultrapassado. Esta propriedade não estará presente na matemática infinitesimal.

Yos', por outro lado, construiu os números hiper-reais como uma extensão fechada do corpo ordenado dos números reais, contendo infinitésimos. Além disso, introduziu uma versão para o Princípio da Transferência de Leibniz rigorosa e compatível com essa extensão.

Essas contribuições seriam essenciais na construção de uma nova análise e de novas propostas para o desenvolvimento do Cálculo Diferencial e Integral com o uso do infinitésimo.

Abraham Robinson, em 1961, apresenta, nos Proceedings of the Royal Academy of Sciences of Amsterdam, nova teoria para a análise matemática, fundamentada nos infinitésimos e usando teoria de modelos: a ANS (ROBINSON, 1996). A ANS corresponde a uma moderna teoria do contínuo, baseada em uma extensão própria do conjunto $\mathrm{N}$ dos números naturais - $\mathrm{N}^{*}$-, e uma extensão própria, não arquimediana, do corpo $\mathrm{R}$ dos números reais - $\mathrm{R}^{*}$. A abordagem modelo-teorética adotada por Robinson (1961), envolvendo estruturas complexas como os ultrafiltros e uma linguagem de $1^{a}$ ordem com restrições adequadas ao tratamento lógico-matemático de $\mathrm{N}^{*}$ e $\mathrm{R}^{*}$, está no foco dos questionamentos e dúvidas com que, como toda teoria inovadora, a ANS foi recebida pela comunidade científica.

Visando adequar essa nova análise ao ensino do Cálculo fundamental, Howard Jerome Keisler (1976), da Universidade de Wisconsin, simplificou sua axiomática e excluiu o uso da lógica para produzir o livro-texto intitulado Elementary calculus: an infinitesimal approach, que foi usado de forma experimental, na primeira metade dos anos 1970, na Universidade de Wisconsin e em algumas outras universidades e faculdades americanas. A experiência, mesmo não sendo levada à frente, como era o desejo de Keisler, apresentou resultados animadores que, ainda hoje, despertam o interesse de educadores e pesquisadores. Mas seu tratamento da ANS também encontraria opositores.

Errett Bishop (1977, p. 206), um de seus maiores críticos, considera que o livro de Keisler (1976) "não oferece evidência de que os números hiper-reais são apenas um expediente para a demonstração de teoremas sobre os números reais". E assim se refere ao que considera "complicações técnicas" introduzidas na obra:

The technical complications introduced by Keisler's approach are of minor importance. The real damage lies in his obfuscation and devitalization of those wonderful ideas. No invocation of Newton and Leibniz is going to justify developing calculus using axioms $\mathrm{V}^{*}$ and $\mathrm{VI}^{*}$ on the grounds that the usual definition of a limit is too complicated! Although it seems to be futile, I always tell my calculus students that mathematics is not esoteric: It is common sense. (Even the notorious $\mathcal{\varepsilon}$, 
$\delta$ definition of limit is common sense, and moreover is central to the important practical problems of approximation and estimation.) They do not believe me. In fact the idea makes them uncomfortable because it contradicts their previous experience. Now we have a calculus text that can be used to confirm their experience of mathematics as an esoteric and meaningless exercise in technique. (BISHOP, 1977, p. 208)

Bishop (1977) questiona os axiomas introduzidos por Keisler (1976), que considera pouco intuitivos, como os axiomas $\mathrm{V}^{*} \mathrm{e} \mathrm{VI}^{*}$, assim enumerados e enunciados:

Axiom D (Function Axiom). For each real function $\mathrm{f}$ of $\mathrm{n}$ variables there is a corresponding hyperreal function $\mathrm{f}^{*}$ of $\mathrm{n}$ variables, called the natural extension of $f$. The field operations of $R^{*}$ are the natural extensions of the field operations of $\mathrm{R}$.

Axiom E (Transfer Axiom). Given two systems of formulas S; T with the same variables, if every real solution of $\mathrm{S}$ is a solution of $\mathrm{T}$, then every hyperreal solution of $\mathrm{S}$ is a solution of T. (KEISLER, 1976, p. 9)

De fato, podemos inferir de Henson e Keisler (1986, p. 377) que a insuficiente compreensão da teoria axiomática da ANS, que apresenta resultados mais fortes do que os da análise standard - caso do Axioma E, que interpretamos na página seguinte -, é a causa mais provável das críticas que recebe.

Paralelamente aos esforços de Keisler (1976) na busca por uma simplificação da linguagem da ANS, considerada por seu próprio criador, Abraham Robinson, uma conveniência surge em 1977, introduzida por Nelson (1977), a Teoria Interna de Conjuntos, ou TIC (originalmente, IST, abreviatura de Internal Set Theory: a new approach to nonstandard analysis). A abordagem sintática de Nelson (1977) é considerada mais elegante e exige menos lógica e teoria de modelos, mas requer um cuidado especial, relacionado com o princípio da formação de conjuntos (ou axioma da compreensão), uma vez que não existem conjuntos na TIC cujos elementos sejam precisamente os números inteiros usuais.

Como inicialmente dito, concluiremos este trabalho enunciando, para o cálculo diferencial paraconsistente, o equivalente ao princípio de transferência, assim enunciado na TIC de Nelson (1977, p. 1174, tradução nossa):

Seja P uma fórmula interna com as seguintes (e únicas) variáveis livres $\mathrm{x}, \mathrm{t}_{1}, \ldots, \mathrm{t}_{\mathrm{n}}$. Então, $8^{\text {st }} t_{1}, \ldots, 8^{\text {st }} t_{n}\left(8^{\text {st }} x P\left(x ; t_{1}, \ldots, t_{n}\right)=>8 x P\left(x ; t_{1}, \ldots, t_{n}\right)\right)$.

O princípio de transferência é um teorema metamatemático, pois quantifica sobre sentenças, estabelecendo que qualquer proposição de primeira ordem, no domínio dos números reais, é logicamente equivalente a uma proposição sintaticamente similar no domínio dos números hiper-reais. Além de colaborar no processo de discussão sobre o modelo ideal para o estudo do cálculo, este princípio ainda assegura que a ANS, da mesma forma como ocorrerá com o cálculo paraconsistente, atua de forma equivalente sobre as proposições clássicas. Com muitas vantagens se considerarmos suas propriedades associadas aos hiper-reais. Cabe adver- 
tir, porém, que há certa sutileza envolvendo este teorema, no que concerne à propriedade arquimediana: o conjunto dos hiper-reais aparenta ser arquimediano para um observador localizado no espaço não-standard, mas não arquimediano para um observador fora desse espaço.

Outros modelos para a ANS surgiram desde então, como, por exemplo, a ANS construtiva de Palmgrem (1995). Sua axiomática se assemelha à TIC de Nelson, mantendo os axiomas (T) e (I), mas substituindo (S) (standardization) pelo conceito dito underspill (se uma proposição é válida, no modelo não-standard, para todos os elementos não-standard, então deve haver pelo menos um elemento standard para o qual a mesma é válida).

Ao mesmo tempo em que se multiplicavam as pesquisas sobre a ANS, buscando sua simplificação, Newton Carneiro Afonso da Costa (COSTA, 2000) propunha a construção de um cálculo diferencial paraconsistente sobre a teoria paraconsistente de conjuntos, tendo o cálculo de predicados paraconsistente de primeira ordem com igualdade $\mathrm{C}_{1}{ }^{=}$como lógica subjacente.

Nessa perspectiva, Costa (2000) propõe o cálculo paraconsistente como uma teoria inconsistente, porém não trivial, satisfazendo o chamado princípio de l'hospital, segundo o qual "duas quantidades distintas, que diferem entre si por não mais do que uma quantidade infinitamente pequena, podem ser consideradas iguais” (L'HOSPITAL, 1696, p. 2, tradução nossa). Assim, em sua essência, os trabalhos de Robinson $(1961,1996)$ e de Costa (2000) evidenciam a importância de se estabelecer, em bases lógicas e conjuntistas rigorosas, a linguagem sobre a qual a análise deve ser desenvolvida, com o uso de infinitésimos.

Informalmente, como observamos para a ANS, há um princípio de transferência estabelecendo, entre o cálculo paraconsistente e o cálculo clássico, que toda proposição válida no primeiro é também válida no segundo, e reciprocamente (CARVALHO, 2004). Esse teorema, além de mostrar sua compatibilidade com o cálculo clássico, estabelece sua compatibilidade com a análise de Robinson $(1961,1996)$. De certo modo, qualquer uma delas poderia ser usada para se demonstrar um teorema da matemática usual.

Não incluímos, aqui, a construção dos objetos da ANS, o que não é nosso objetivo, mas estes podem ser encontrados em inúmeros textos, alguns dos quais indicados nas Referências, ao final deste trabalho.

\section{$\mathrm{O}$ cálculo paraconsistente de predicados $\mathrm{C}_{1}=$ e a teoria de conjuntos $\mathrm{CHU}_{1}$ de Costa}

Newton da Costa foi o brasileiro responsável pela introdução do primeiro e mais importante sistema lógico distinto da lógica clássica $\left(C_{n}=, 1<n<\omega\right)$, incorporando o cálculo proposicional e o cálculo de predicados, internacionalmente reconhecido como tal. Também introduziu uma hierarquia de teorias paraconsistentes de conjuntos $\left(\mathrm{CHU}_{n}, 1<\mathrm{n}<\omega\right)$, motivado pela teoria de conjuntos clássica CHU (CHURCH, 1940; COSTA, 1974, 1986, 1993; D’OTTAVIANO, 1992). Baseado na teoria clássica de conjuntos ZF - que conhecemos de alguma forma por sua presença nas atividades matemáticas que desenvolvemos quando lidamos com operações e propriedades de conjuntos - Costa (2000) introduz e Carvalho (2004, p. 71-80) desenvolve propriedades do "anel dos números hiper-reais $\uparrow$ " e do "quase-anel dos números hiper-reais estendidos $\mathfrak{A}$ *, aos quais vamos nos referir mais à frente. Aqui a ideia de 
anel é a que conhecemos da álgebra estudada nos cursos de graduação em matemática. Como é comum a todas as áreas de formação, quando avançamos nas teorias, novos objetos cruzam nossos caminhos e surge, então, a necessidade de nos abrirmos para a expansão de sua linguagem e a compreensão de conceitos nem sempre muito intuitivos mas, quase sempre, mais simples do que aparentam ser.

Faremos, aqui, uma introdução informal e abreviada dos demais objetos que compõem o Cálculo paraconsistente. As estruturas algébricas $A$ e $A^{*}$ são extensões do corpo $R$ dos números reais standard, e os elementos de $A$ e $\AA^{*}$ são chamados, respectivamente, de números hiper-reais e números hiper-reais generalizados, ou simplesmente g-reais. Baseado em $\boldsymbol{A}^{*}$, Costa (2000) propõe a construção do cálculo diferencial paraconsistente $\mathbb{P}$, cuja linguagem é a linguagem $\mathrm{L}^{=}$do sistema $\mathrm{C}_{1}{ }^{=}$e cuja teoria de conjuntos subjacentes é $\mathrm{CHU}_{1}$ estendida à linguagem de $\mathrm{CHU}_{1}$, nas quais lidamos com os elementos de $\mathfrak{A}$ *

Baseados em Costa $(1974,1986,1993,2000)$, introduzimos definições generalizadas para diversos conceitos e provamos diversos teoremas que generalizam importantes resultados clássicos, e apresentamos algumas aplicações desses resultados.

Costa (2000) introduz os conceitos de superestrutura paraconsistente sobre um conjunto $\mathrm{X}$ de átomos de $\mathrm{CHU}_{1}$, e de monomorfismo entre superestruturas paraconsistentes, com base em Robinson e Zakon (1969), e Stroyan e Luxemburg (1976).

Apresentamos, a seguir, alguns de seus objetos mais relevantes, sem maiores considerações formais.

A linguagem $L_{0}=\mathrm{da}$ lógica utilizada inclui os conectivos $\neg, \wedge, \vee, \mathrm{e} \supset(\mathrm{ou} \rightarrow$ ), o símbolo de predicado de igualdade, os quantificadores existencial $(\exists)$ e universal $(\forall)$ e parênteses, mais famílias, predicados e funções (ver da Costa, 1993). As noções de fórmula e teorema, bem como as convenções gerais e notações são as usuais, como em Kleene (1952).

Na definição de fórmula de $\mathrm{L}_{0}{ }^{*}$ temos a cláusula adicional: Se $\mathrm{t}_{1}$ e $\mathrm{t}_{2}$ são termos, então $\mathrm{t}_{1}$ $=\mathrm{t}_{2}$ é uma fórmula de $\mathrm{L}^{=}$.

Sejam A e B duas fórmulas. Usando nossos conhecidos da lógica elementar, podemos interpretar convenientemente os seguintes operadores da linguagem $\mathrm{L}_{0}=$ :

Definição 1. $\mathrm{A}^{\circ}={ }_{\text {def }} \neg(\mathrm{A} \wedge \neg \mathrm{A})$. Lê-se: $\mathrm{A}^{\circ}$, ou "A bola", que indica ser a fórmula "A" "bem comportada". De outra forma, indica que não pode ocorrer "A" e a sua negação $\neg$ A. Caso isso pudesse ocorrer, estaríamos frente a uma situação paraconsistente. "A" poderia representar no Direito, por exemplo, uma situação de culpa e de inocência, ao mesmo tempo, sem entrar no mérito do que se faria a respeito.

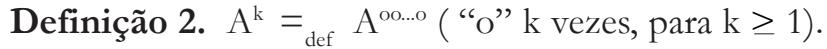

Definição 3. $A^{(k)}=\operatorname{def} A^{1} \wedge \ldots \wedge A^{k}$, para $k \geq 1$.

Definição 4. $\neg^{*} \mathrm{~A}={ }_{\text {def }} \neg \mathrm{A} \wedge \mathrm{A}^{\circ}$.

Definição 5. $\sim_{k} A={ }_{\text {def }} \neg A \wedge A^{(k)}$, para $k \geq 1$

Definição 6. $(\mathrm{A} \leftrightarrow \mathrm{B})=_{\text {def }}(\mathrm{A} \supset \mathrm{B}) \wedge(\mathrm{B} \supset \mathrm{A})$.

A negação " $\neg$ ” é a negação básica do sistema $C_{1}$, usualmente denominada "negação fraca". A negação " $\neg$ ", denominada "negação forte", desempenha, nesse sistema, papel equivalente ao da negação clássica. Lê-se $\neg$ A como "negação de A" ou "negação fraca de A", e $\neg$ 'A como "negação forte de A". 
Regra 1. $\mathrm{A}, \mathrm{A} \supset \mathrm{B}$, Regra de Modus Ponens (MP)

$\mathrm{B}$

Regra 2. $\frac{A \rightarrow B(x)}{A \rightarrow \forall \times B(x)}$, onde x não ocorre livre em A.

Regra 3. $\frac{A(x) \rightarrow B}{\exists x A x \rightarrow B}$, onde $x$ não ocorre livre em $B$.

Omitiremos, para favorecer a sequência da leitura, as propriedades da teoria de conjuntos $\mathrm{CHU}_{1}$, que estará subentendida daqui à frente. Conscientemente, sacrificaremos o detalhamento das propriedades dos objetos que caracterizam o cálculo paraconsistente, em favor de sua maior abrangência, acreditando que os interessados em se aprofundar no assunto possam recorrer a fontes adequadas para isso, algumas das quais apontadas nas Referências.

\section{Do cálculo não-standard ao cálculo diferencial paraconsistente}

Intuitivamente, um infinitésimo é um número que é menor do que qualquer número real positivo e é maior do que cada número real negativo, ou, equivalentemente, em valor absoluto é menor do que $1 / \mathrm{m}$ para todo m inteiro positivo. Zero é o único número real que, ao mesmo tempo, é um infinitesimal. Números infinitesimais diferentes de zero não ocorrem na matemática clássica. Ainda, eles podem ser tratados e se comportam, em boa parte, da mesma forma como são tratados e se comportam os números reais clássicos.

Na construção de Costa (2000) para o Cálculo Paraconsistente, a extensão A do corpo $\mathrm{R}$ dos números reais constitui um contínuo não arquimediano sem números infinitos, ao passo que a extensão $\mathfrak{A}^{*}$ do mesmo corpo constitui um contínuo não arquimediano, com infinitésimos e infinitos.

\section{$O$ anel $\uparrow$ dos números hiper-reais}

Sejam I um intervalo real fixado e a um elemento do interior de I.

Definição $7^{3 .}$ Uma variável infinitesimal é uma função real $\mathrm{f}: \mathrm{I} \subseteq \mathrm{R} \rightarrow \mathrm{R}$, tal que $\mathrm{f}(\mathrm{x})=0$. Denotamos o conjunto das variáveis infinitesimais por $V$.

Definição 8. O conjunto dos números hiper-reais, denotado por $\AA$, é assim definido: $\boldsymbol{t}={ }_{\text {def }}\{\langle r, f\rangle: r \in \mathbb{R}$ e $f \in V\}$.

Os números do conjunto đA são também chamados números reais generalizados, ou, simplesmente, g-reais. Todo número real $\mathrm{r}, \mathrm{r} \in \mathbb{R}$, pode ser identificado com o hiper-real da forma $\langle\mathrm{r}, 0\rangle$, que é dito um número real standard.

\footnotetext{
${ }^{3}$ Igualmente podem ser usados limites laterais na definição das variáveis infinitesimais, observando-se que o conceito de limite aqui utilizado é o clássico.
} 
Definição 9. Um infinitésimo é qualquer número hiper-real da forma $\langle 0, \mathrm{f}\rangle$, em que $\mathrm{f}$ é uma variável infinitesimal. Para cada $r \in \mathbb{R}$, o conjunto dos hiper-reais da forma $\langle r, f\rangle$ é dito mônada de $\mathrm{r}$, denotado por [r]. A mônada do zero é o conjunto constituído pelos infinitésimos.

Definição 10. A igualdade, ou identidade, de dois números hiper-reais de $\uparrow$, denotada por "=", é definida por: $\langle\mathrm{r}, \mathrm{f}\rangle=\langle\mathrm{s}, \mathrm{g}\rangle$ se, e somente se, $\mathrm{r}=\mathrm{s}$ e $\mathrm{f}=\mathrm{g}$.

Definição 11. A adição $(+)$ e a multiplicação $(X)$ de dois números hiper-reais de $\uparrow$ são definidas por:

(i) $\langle\mathrm{r}, \mathrm{f}\rangle+\langle\mathrm{s}, \mathrm{g}\rangle={ }_{\text {def }}\langle\mathrm{r}+\mathrm{s}, \mathrm{f}+\mathrm{g}\rangle ; \quad$ (ii) $\langle\mathrm{r}, \mathrm{f}\rangle \times\langle\mathrm{s}, \mathrm{g}\rangle={ }_{\text {def }}\langle\mathrm{rs}, \mathrm{rg}+\mathrm{fs}+\mathrm{fg}\rangle$.

Para qualquer hiper-real $\langle\mathrm{r}, \mathrm{f}\rangle$, podemos escrever: $\langle\mathrm{r}, \mathrm{f}\rangle=\langle\mathrm{r}, 0\rangle+\langle 0, \mathrm{f}\rangle$.

Teorema 1. A estrutura $\langle\uparrow,+, \times, 0,1\rangle$, em que " 0 " e " 1 " representam, respectivamente, o hiper-real nulo $\langle 0,0\rangle$ e o hiper-real $\langle 1,0\rangle$, é um anel comutativo com unidade.

A estrutura de corpo $\langle\mathrm{R},+, ., 0,1\rangle$ pode ser vista como um subanel de $\langle\AA,+, \times, 0,1\rangle$, pela identificação de todo par da forma $\langle\mathrm{r}, 0\rangle$ com o número real $\mathrm{r}$. A relação de ordem $<$, de $\mathbb{R}$, pode ser estendida a $\boldsymbol{A}$, onde é não linear.

Definição 12. $\langle\mathrm{r}, \mathrm{f}\rangle<\langle\mathrm{s}, \mathrm{g}\rangle$ se, e somente se, $\mathrm{r}<\mathrm{s}$, ou $\mathrm{r}=\mathrm{s}$ e $\mathrm{f}(\mathrm{x})<\mathrm{g}(\mathrm{x}), \forall \mathrm{x} \in \mathrm{I}$.

Definição 13. A ordem de um infinitésimo qualquer, $\langle 0, \mathrm{~g}\rangle$, relativamente a um infinitésimo $\langle 0, \mathrm{f}\rangle$, é definida por:

a) $\langle 0, f\rangle$ e $\langle 0, g\rangle$ possuem mesma ordem se $\lim _{x \rightarrow a} \frac{f(x)}{g(x)}=b$, sendo b um número real standard diferente de zero;

b) A ordem de $\langle 0, f\rangle$ é superior à de $\langle 0, g\rangle$ se $\lim _{x \rightarrow a} \frac{f(x)}{g(x)}=0$;

c) $\langle 0, \mathrm{f}\rangle$ é de ordem $\mathrm{k}$ relativamente a $\langle 0, \mathrm{~g}\rangle$ se $\lim _{\mathrm{x} \rightarrow \mathrm{a}} \frac{\mathrm{f}(\mathrm{x})}{[\mathrm{g}(\mathrm{x})]^{\mathrm{k}}}=\mathrm{b}$, para b um número real standard diferente de zero.

Dois infinitésimos $\langle 0, \mathrm{f}\rangle \mathrm{e}\langle 0, \mathrm{~g}\rangle$ são ditos equivalentes quando $\lim _{\mathrm{x} \rightarrow \mathrm{a}} \frac{\mathrm{f}(\mathrm{x})}{\mathrm{g}(\mathrm{x})}=1$.

Dada uma função $f$ qualquer, definida em $R$, esta pode ser facilmente estendida a uma hiperfunção $f: A \rightarrow \uparrow$. E dado um número hiper-real $r \in A$, como $r=\left\langle r^{\prime}, g\right\rangle=\left\langle r^{\prime}, 0\right\rangle+$ $\langle 0, g\rangle$, podemos escrever $f(r)=f\left(r^{\prime}+\varepsilon\right)$, sendo $f$, portanto, uma função de função de $\varepsilon$. Assim, podemos expressar naturalmente a noção de limite da análise clássica na linguagem dos infinitésimos.

Definição 14. Dada uma função hiper-real $\mathrm{f}: \mathrm{B} \subseteq \AA \rightarrow A$, $\lim _{x \rightarrow r} f(x)=b$ se, e somente se, $x \in[r]$ implica que $f(x) \in[b]$.

Definição 15. Uma variável infinita é uma função $v, v: I \subseteq R \rightarrow R$, tal que $\lim _{x \rightarrow a} v(x)=\infty$.

Definição 16. Um número hiper-real infinito é um par da forma $\langle v, 0\rangle$, em que v é uma variável infinita.

Definição 17. O conjunto dos números hiper-reais estendidos, denotado por $\overbrace{}^{*}$, é definido por: $\mathbb{A}^{*}=\{\mathrm{a}: \mathrm{a} \in \mathbb{A}$ ou a é um hiper-real infinito $\}$. 
Calculus infinitesimalis: uma teoria ...

As operações de adição e multiplicação e a relação de igualdade (identidade) de $\uparrow$ podem ser estendidas a $\boldsymbol{A}^{*}$, de tal modo que a estrutura $\left\langle\boldsymbol{A}^{*},+, \times, 0,1\right\rangle$ conserve algumas das propriedades algébricas importantes do hiperanel $\langle\uparrow,+, \times, 0,1\rangle$. Como, porém, falham algumas cláusulas da definição de anel, nós a denominamos um quase-anel. Através de ti* é que delineamos uma teoria paraconsistente para o cálculo diferencial.

Aplicação 1. A aceleração no movimento circular uniforme (aceleração centrípeta)

Consideremos uma partícula que descreve um movimento circular de raio $\mathrm{r}$, em torno de um ponto $\mathrm{O}$, pela ação de uma força centrada no mesmo. Consideremos, também, que no instante $t$ a partícula se encontre na posição $P$ da figura abaixo, dada por um ângulo $\alpha$ formado entre o raio e o eixo $\mathrm{x}$, e coordenadas $(\mathrm{x}, \mathrm{y})$, todos dependendo de $\mathrm{t}$ :

$\alpha=\alpha(t), x=x(t), e y=y(t)$.

Desejamos estabelecer uma fórmula para a aceleração da partícula, usando algumas das propriedades do quase anel $\boldsymbol{A}^{*}$. Sob uma variação infinitesimal $\theta>0$ ( $\theta$ inversível $)$ do tempo t, a partícula sofrerá uma alteração em sua posição, passando do ponto $\mathrm{P}$ a um ponto $\mathrm{Q}$, determinado por um raio, formando com o eixo $x$, um ângulo $\alpha(t+\theta)$. $\mathrm{O}$ acréscimo angular será, pois, $\varepsilon=\alpha(t+\theta)-\alpha(t)$ (se julgar necessário, faça uma figura para acompanhar o desenvolvimento da construção). Fatos que podemos assumir:

a) Se $\mathrm{x} \equiv 0$, sendo a variável infinitesimal associada a x comparável com a função nula 0 , então senx $\equiv \mathrm{x}$. Mas, sendo $\mathrm{x} \equiv 0$, podemos escrever $\mathrm{x}=\gamma, \gamma$ infinitesimal. Valendo, sob a restrição inicial para $\mathrm{x}$, a relação $|\operatorname{senx}| \leq|\mathrm{x}| \operatorname{em} \boldsymbol{A}^{*}$, temos que $|\operatorname{senx}|=|\operatorname{sen}(\gamma)| \leq|\gamma|$. Como $0<\mathrm{x}<\pi / 2$, podemos escrever $0<\operatorname{sen} \gamma<\gamma$, concluindo que sen $\gamma \equiv \gamma$.

b) $\mathrm{f}(\mathrm{r}+\varepsilon)-\mathrm{f}(\mathrm{r})=\mathrm{P}(\mathrm{r}) \times \varepsilon+\delta_{1}$, sendo $\delta_{1}$ infinitésimo de ordem superior à de $\varepsilon$, para todo $\varepsilon$ infinitesimal.

Posicionando os vetores-velocidade $\overrightarrow{v_{p}}$ e $\overrightarrow{v_{q}}$ da partícula em P e Q numa origem comum, $\overline{\mathrm{O}}$, comparando com os vetores-posição em $\stackrel{q}{\mathrm{P}}$ e Q, temos, com o uso das propriedades que acabamos de assinalar, os vetores-velocidade:
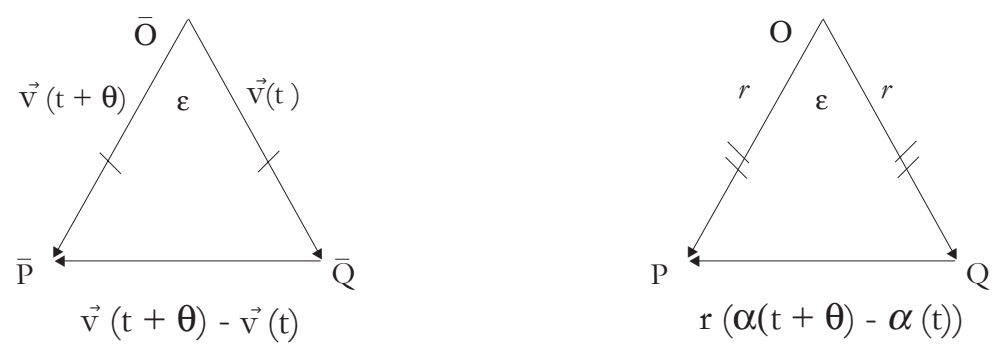

Os vetores-velocidade são perpendiculares aos raios, na direção dos quais os vetoresaceleração apontam para o centro $\mathrm{O}$. Como as equações $\mathrm{x}, \mathrm{y}, \alpha$ e $\mathrm{v}$ são diferenciáveis em $\mathrm{t}$, temos por (b) e pela relação $\equiv$ em $\boldsymbol{t}^{*}$, que $\alpha(\mathrm{t}+\theta)-\alpha(\mathrm{t}) \equiv \alpha^{\prime}(\mathrm{t}) \times \theta$ e v(t $\left.+\theta\right)-\mathrm{v}(\mathrm{t}) \equiv \mathrm{v}^{\prime}(\mathrm{t})$ $\times \theta$ (as duas expressões diferem por um infinitésimo de ordem superior à de $\theta$ ). 
Como os triângulos OPQ e $\overline{\mathrm{O}} \overline{\mathrm{P}} \overline{\mathrm{Q}}$ são semelhantes, temos:

$$
\begin{aligned}
& \frac{\mathrm{v}(\mathrm{t}+\theta)-\mathrm{v}(\mathrm{t})}{\mathrm{v}(\mathrm{t})} \equiv \frac{\mathrm{r}(\alpha(\mathrm{t}+\theta)-\alpha(\mathrm{t}))}{\mathrm{r}} \mathrm{e} \\
& \frac{\mathrm{v}^{\prime}(\mathrm{t}) \times \theta}{\mathrm{v}(\mathrm{t})} \equiv \frac{\mathrm{r}(\alpha(\mathrm{t}+\theta)-\alpha(\mathrm{t}))}{\mathrm{r}} \rightarrow \mathrm{v}^{\prime}(\mathrm{t}) \equiv \underbrace{\underbrace{\underbrace{}_{0}}_{\mathrm{r}(\alpha(\mathrm{t}+\theta)-\alpha(\mathrm{t}))}}_{\equiv \mathrm{v}(\mathrm{t})} \times \frac{\mathrm{v}(\mathrm{t})}{\mathrm{r}}
\end{aligned}
$$

$\operatorname{Logo}, \mathrm{v}^{\prime}(\mathrm{t}) \equiv \frac{\mathrm{v}^{2}(\mathrm{t})}{\mathrm{r}}$, que é a lei procurada para a aceleração.

\section{O cálculo diferencial paraconsistente}

Costa (2000) introduz o cálculo diferencial paraconsistente Ç, apresentando em detalhes os conceitos que aparecem à frente. Este cálculo é interpretado na estrutura clássica $\AA^{*}$, como acima visto, contendo tanto números infinitesimais quanto números infinitos. Em uma linguagem lógica rigorosa, temos, conforme esse autor, que este cálculo é não trivial - mas inconsistente -, se a análise clássica for consistente. Dito de outra forma, quem o garante é a própria análise clássica: se esta, de fato, é uma teoria livre de propriedades capazes de invalidála, então, embora associado a propriedades inconsistentes - que não o invalidam, pois este se submete à lógica paraconsistente que admite inconsistências -, este cálculo também estará livre de tais propriedades, digamos, catastróficas.

A linguagem $\mathrm{L}$ de $\mathrm{CDp}$ é a linguagem $\mathrm{L}^{=}$de $\mathrm{C}_{1}{ }$, estendida à linguagem de $\mathrm{CHU}_{1}$, com símbolos funcionais; constantes especiais para nomear os indivíduos da estrutura $\mathrm{A}^{*}$; o predicado <; as operações de $\boldsymbol{A}^{*}$; e três espécies de variáveis individuais, para denotarem, respectivamente, hiper-reais finitos - $r, s, \ldots-$, infinitésimos $-\delta, \varepsilon, \ldots-$, e infinitos.

A lógica subjacente a CDp é o cálculo paraconsistente de predicados de primeira ordem com igualdade $\mathrm{C}_{1}{ }^{=}$, e a teoria de conjuntos subjacente é a teoria paraconsistente de conjuntos $\mathrm{CHU}_{1}$ de da Costa (2000).

Introduzimos em L, a seguir, por definição, o predicado “ $\equiv$ “ que representa um predicado de igualdade generalizado, necessário para a comparação de números que diferem entre si apenas infinitesimalmente.

Definição 18. O predicado de igualdade generalizada, ou identidade generalizada, denotado por " $\equiv$ ", é definido por: $\mathrm{t}_{1} \equiv \mathrm{t}_{2}={ }_{\text {def }} \mathrm{t}_{1}-\mathrm{t}_{2}=\varepsilon$, com $\mathrm{t}_{1}$ e $\mathrm{t}_{2}$ termos da linguagem, $\varepsilon$ infinitésimo, $\mathrm{e}=$ o predicado primitivo de igualdade de $\mathrm{L}$.

Definimos $\neg\left(\mathrm{t}_{1} \equiv \mathrm{t}_{2}\right)$, o que denotamos por $\mathrm{t}_{1} \mathrm{~T} \mathrm{t}_{2}$, por: $\mathrm{t}_{1} \mathrm{~T} \mathrm{t}_{2}==_{\text {def }} \mathrm{t}_{1} \neq \mathrm{t}_{2}$.

Definição 19. Uma função hiper-real (ou hiper-função) é uma função f cujo domínio é um subconjunto do conjunto $\AA$ de hiper-reais, com valores em $\mathfrak{A}^{*}: \mathrm{f}: \mathrm{B} \subseteq \boldsymbol{A} \rightarrow \boldsymbol{A}^{*}$.

A definição de limite de uma função hiper-real $\mathrm{f}(\mathrm{x})$, quando $\mathrm{x}$ tende a um número real standard, é a usual.

Definição 20. Dada uma função hiper-real $f: B \subseteq A \rightarrow A^{*}$ e números reais standard r e b, temos: 
$\lim _{x \rightarrow r} f(x)=b$

se, e somente se, $(\forall \varepsilon>0)(\exists \delta>0)(\forall \mathrm{x})(0<|\mathrm{x}-\mathrm{r}|<\delta \rightarrow|\mathrm{f}(\mathrm{x})-\mathrm{b}|<\varepsilon$.

Teorema 2. Dada uma função hiper-real $\mathrm{f}: \mathrm{B} \subseteq \mathfrak{A} \rightarrow \mathfrak{A}^{*}$, temos que: $\lim _{\mathrm{x} \rightarrow \mathrm{r}} \mathrm{f}(\mathrm{x})=\mathrm{k}$ se, e somente se, $(\forall x)(x \in B)((x \equiv r) \rightarrow(f(x) \equiv k))$.

Podemos introduzir, na linguagem L, os conceitos de limite de uma função hiper-real nos casos em que $\mathrm{x}$, ou $\mathrm{f}(\mathrm{x})$, tendem ao infinito.

Definição 21. Dada uma função hiper-real $\mathrm{f}: \mathrm{B} \subseteq A \rightarrow A^{*}$ e um real standard $\mathrm{b}$, temos: $\lim \mathrm{f}(\mathrm{x})=\mathrm{b}$

$\mathrm{x} \rightarrow\langle\mathrm{u}, 0\rangle$

se, e somente se, $\left(\exists\langle\mathrm{v}, 0\rangle \in \mathfrak{A}^{*}\right)(\forall \mathrm{x})((|\mathrm{x}|>|\langle\mathrm{v}, 0\rangle|) \rightarrow(\mathrm{f}(\mathrm{x}) \equiv \mathrm{b}))$.

Os outros casos de limites infinitos são definidos analogamente.

Definição 22. Uma função hiper-real $\mathrm{f}: \mathrm{U} \subseteq \uparrow \rightarrow A^{*}$ é contínua em um hiper-real $\langle\mathrm{r}, \mathrm{g}\rangle \in \mathrm{U}$ se, e somente se $(\forall\langle\mathrm{s}, \mathrm{h}\rangle \in \mathrm{U})(\langle\mathrm{r}, \mathrm{g}\rangle \equiv\langle\mathrm{s}, \mathrm{h}\rangle \rightarrow \mathrm{f}(\langle\mathrm{r}, \mathrm{g}\rangle) \equiv \mathrm{f}(\langle\mathrm{s}, \mathrm{h}\rangle)$. Caso contrário, $\mathrm{f}$ é descontínua em $\langle\mathrm{r}, \mathrm{g}\rangle$.

Definição 23. A derivada de uma função hiper-real $f: A \rightarrow A^{*}$, em um número real standard $\mathrm{r}$, denotada por $\mathrm{f}(\mathrm{r})$, é um número real standard, denotado por $\mathrm{f}(\mathrm{r})$, tal que

$\mathrm{f}(\mathrm{r}+\varepsilon)-\mathrm{f}(\mathrm{r})=\mathrm{f}^{\prime}(\mathrm{r}) \times \varepsilon+\mathrm{d}$,

onde $\varepsilon$ é um infinitésimo arbitrário, e $\delta$ é um infinitésimo com ordem superior à de $\varepsilon$, do qual depende.

Observação 1. De acordo com a definição, $D$ será o valor da derivada de $\mathrm{f}$ em um número real standard $\mathrm{r}$, isto é, $\mathrm{D}=\mathrm{f}^{\prime}(\mathrm{r})$, se tivermos $\mathrm{f}(\mathrm{r}+\varepsilon)-\mathrm{f}(\mathrm{r}) \equiv \mathrm{D} \times \varepsilon$.

As operações de derivação e os teoremas principais do cálculo clássico usual preservam suas características no cálculo paraconsistente, no qual podem ser demonstrados teoremas clássicos, como: o Teorema de Weierstrass, Teorema de Rolle, Teorema do Valor Intermediário e Teorema do Valor Médio. Baseados em trabalhos de Robinson (1996), Robinson e Zakon (1969) e Stroyan e Luxemburg (1976), desenvolvemos, para o Cálculo Diferencial Paraconsistente, um Teorema de Transferência que estabelece as condições sob as quais essa compatibilidade ocorre. Apresentamos, a seguir, o seu enunciado.

\section{Teorema 3. Teorema de transferência}

Sejam as superestruturas $R$ e $\odot$, as funções de interpretação i e i”, e o monomorfismo -, como introduzidos acima, e seja $\alpha\left(\mathrm{x}_{1}, \mathrm{x}_{2}, \ldots, \mathrm{x}_{\mathrm{n}}\right)$ uma fórmula de L, cujas variáveis livres estão entre $\mathrm{x}_{1}, \mathrm{x}_{2}, \ldots, \mathrm{x}_{\mathrm{n}}$. Nessas condições, $\alpha\left(\mathrm{x}_{1}, \mathrm{x}_{2}, \ldots, \mathrm{x}_{\mathrm{n}}\right)$ é válida em $R$ relativamente a $v_{\mathrm{i}}$ se, e somente se, $\bullet\left(\alpha\left(\mathrm{x}_{1}, \mathrm{x}_{2}, \ldots, \mathrm{x}_{\mathrm{n}}\right)\right)$ é válida em $\mathrm{S}$ relativamente a $v_{\mathrm{i}}$, .

\section{Considerações finais}

O cálculo infinitesimal ocupa, na história da Ciência Moderna, um capítulo de profundo significado que, infelizmente, carece de um espaço mais amplo no capítulo de formação básica do currículo dos cursos de graduação, como os cursos de Engenharia, em suas diversas modalidades, e, mesmo, cursos de Licenciatura, como os de Matemática, Física, Pedagogia e 
Filosofia. Perde-se, sem esse espaço, a oportunidade de se conhecer melhor o seu real significado, sua história, o processo de criação e consolidação da linguagem científica e, particularmente, a consciência de que o cálculo diferencial e integral contemporâneo é parte de uma teoria em expansão, cujos limites mal podemos imaginar. A esse respeito, Longo (1999, 2005) busca conexões entre os conceitos matemáticos - pilares do conhecimento, da objetividade e da estabilidade conceitual, que interage fortemente na constituição dos objetos -, a física e a genealogia dos conceitos em outras ciências, como a biologia e a história. Observando, por outro lado, o contexto atual e globalizado do ensino do cálculo, a partir da produção científica que trata, sob variados ângulos, desse tema, verificamos que predomina a busca pela forma ideal para tornar mais acessível e prático o seu ensino. Fato compreensível e desejável, mas que não deveria excluir atividades e enfoques históricos e epistemológicos, que só fariam enriquecer a formação de cada aluno. Algo como sugere o trabalho desenvolvido na Universidade Estadual do Rio Grande do Sul (UERGS), pelos professores Tânia Cristina Baptista Cabral e Roberto Ribeiro Baldino, relacionado com o uso do cálculo infinitesimal no curso de Engenharia em Sistemas Digitais (CABRAL; BALDINO, 2006).

Fleuriot e Paulson (1998) também ilustram a potencialidade dos infinitésimos em aplicações da ANS da geometria à Física de Newton. Arkeryd (2004), físico polonês, faz uso da ANS para o estudo de partículas de gases rarefeitos, submetidos a forças infinitas, como a força gravitacional, estranhando que os próprios matemáticos não desenvolvam mais pesquisas utilizando o cálculo infinitesimal. Curiosamente, Arkeryd (2004), nesse trabalho, nos remete, mais uma vez, ao passado, lembrando que Anaximenes de Mileto elegeu exatamente o ar como elemento primordial, no que, bem mais do que coincidência, sentimos a presença da linha temporal e causal que une o conhecimento científico de todas as épocas.

O trabalho inclui considerações sobre uma lógica pouco divulgada ainda, a lógica paraconsistente. Sobre isso há uma questão que vagou pelo universo científico por muitos séculos: a lógica clássica é a única lógica possível? Mais uma vez, nos reportamos à física, ou à física contemporânea, cujos fenômenos colocam essa questão em primeiro plano. Nem sempre algo se alinha precisamente com a verdade ou com a falsidade. Essa foi uma das motivações de Newton da Costa para sua criação. E a proposta do cálculo paraconsistente foi uma das consequências dessa criação que, antes de pôr em dúvida a importância da lógica de Artistóteles, associa-se à mesma e aos demais sistemas lógicos verdadeiramente relevantes, que fortalecem a ciência como um todo. Concebido com o seu uso, o cálculo diferencial paraconsistente corrobora e amplia a perspectiva anteriormente colocada, mostrando, através dos infinitésimos e da lógica paraconsistente, como teorias sólidas podem ser construídas a partir de elementos que, em sua base, conservam aspectos insólitos, ou aspectos que extrapolam os limites do senso comum. Sua construção faz uso e também amplia aspectos da linguagem científica, da linguagem lógica e da linguagem conjuntista. Da mesma forma como o uso da lógica paraconsistente tem crescido e se diversificado, desde as primeiras pesquisas de da Costa, despertando o interesse da Medicina, do Direito, da Robótica e da Física, acreditamos que o cálculo diferencial paraconsistente se estenderá ao cálculo integral paraconsistente e às equações diferenciais paraconsistentes, compondo, com o cálculo clássico e a ANS, um ferramental matemático capaz de enfrentar, com maior eficiência, rigor lógico-matemático e até simplicidade, problemas matemáticos que não possam ser convenientemente tratados pela análise convencional. 
Calculus infinitesimalis: uma teoria ...

Concluímos observando que o cálculo, como é mais comumente chamado, é, de fato, uma teoria cercada de mitos, que geram a crença em sua dificuldade intrínseca. A crença na impossibilidade de ser aprendido como qualquer outra disciplina da área de matemática, dos cursos de graduação. Ignorar que ajudam a criar e propagar esse mito a baixa compreensão de sua linguagem - cuja construção deve-se dar desde o Ensino Fundamental, incluindo as propriedades elementares do cálculo proposicional e do cálculo de predicados -, a insistência na abordagem utilitarista - que exclui a sua história e muito do que o aproxima da física, da filosofia e de como se constrói o conhecimento científico -, e o uso inconsciente de recursos computacionais, é fechar os olhos para uma clara realidade.

\section{Referências}

ARKERYD, L. Applications of NSA to mathematical physics. Delta, Warsaw, v. 7, n. 362, p. 12-13, 2004.

BARON, M. E. The origins of the infinitesimal calculus. Mineola: Dover Publications, 2004.

BISHOP, E. Review: H. Jerome Keisler, elementary calculus. Bulletin of the American Mathematical Society, Providence, v. 83, n. 2, p. 205-208, 1977.

CABRAL, T. C. B.; BALDINO, R. R. Cálculo infinitesimal para um curso de engenharia. Revista de Ensino de Engenharia, São Paulo, v. 25, n. 1, p. 3-16, 2006.

CARVALHO, T. F. Sobre o cálculo diferencial paraconsistente de da Costa. 2004. 200

f. Tese (Doutorado em Filosofia) - Instituto de Filosofia e Ciências Humanas, Universidade Estadual de Campinas, Campinas, 2004.

CHURCH, A. A formulation of the simple theory of types. The Journal of Symbolic Logic, Poughkeepsie, v. 5, n. 2, p. 56-68, 1940.

COSTA, N. C. A. da. On the theory of inconsistent formal systems. Notre Dame Journal of Formal Logic, Durham, v. 15, n. 4, p. 497-510, 1974.

1986. On paraconsistent set theory. Logique et Analyse, Brussels, v. 115, p. 361-371, Sistemas formais inconsistentes. Curitiba: Ed. UFPR, 1993.

Paraconsistent mathematics. In: WORLD CONGRESS ON

PARACONSISTENCY, 1., 1998, Ghent, Belgium. Frontiers in Paraconsistent Logic: proceedings... London: King's College Publications, 2000. p. 165-179.

D’OTTAVIANO, I. M. L. A lógica clássica e o surgimento das lógicas não clássicas. In: ÉVORA, F. (Ed.). Século XIX: o nascimento da ciência contemporânea. Campinas: Unicamp, 1992. p. 65-93. (Coleção CLE, v. 11).

EVES, H. Introdução à história da matemática. Campinas: Editora Unicamp, 2004. 
FLEURIOT, D. F.; PAULSON, L. C. A combination of nonstandard analysis and geometry theorem proving, with application to Newton's principia. In: INTERNATIONAL CONFERENCE ON AUTOMATED DEDUCTION, 15., 1998. Proceedings ... London: Springer-Verlag, 1998. p. 3-16. (Lecture notes in computer science, 1421).

HEATH, T. The method of Archimedes recently discovered by Heiberg: (a supplement to the works of Archimedes, 1897). Cambridge: Cambridge University Press, 1912.

HENSON, C. W.; KEISLER, H. J. On the strength of nonstandard analysis. The Journal of Symbolic Logic, Poughkeepsie, v. 51, n. 2, p. 377-386, 1986.

KEISLER, H. J. Foundations of infinitesimal calculus. Boston: Prindle Weber \& Schmidt, 1976.

Foundations of infinitesimal calculus. [S.l.: s.n.], 2007. Disponível em: <http://www.math.wisc.edu/ keisler/foundations.html>. Acesso em: 03 set. 2012.

KLEENE, S. C. Introduction to metamathematics. Amsterdam: North Holland; New York: Van Nostrand, 1952.

L'HOSPITAL, G. F. A. Analyse des infiniment petits pour l'intelligence des lignes courbes. Paris: Imprimerie Royale, 1696.

LONGO, G. The mathematical continuum: from intuition to logic. In: PETITOT, J. et al. (Ed.). Naturalizing phenomenology: issues in contemporary phenomenology and cognitive science. Stanford: Stanford University Press, 1999. p. 401-428.

. Mathematical concepts: a constructive approach. In: BOI, L.; KERSZBERG, P.; PATRAS, F. (Ed.). Rediscovering phenomenology: phenomenological essays on mathematical beings, physical reality, perception and consciousness. Utrecht: Kluwer, 2005.

NELSON, E. Internal set theory: a new approach to non-standard analysis. Bulletin of American Mathematical Society, Providence, v. 83, n. 6, p. 1165-1198, 1977.

PALMGREN, E. A constructive approach to nonstandard analysis. Annals of Pure and Applied Logic, Amsterdam, v. 73, p. 297-325, 1995.

ROBINSON, A. Non-standard analysis. Proceedings of the Royal Academy of Sciences, Amsterdam, ser. A, n. 64, p. 432-440, 1961.

. Non-standard analysis. Princeton: Princeton University Press, 1996.

ROBINSON, A.; ZAKON, E. A set theoretical characterization of enlargements. In: LUXEMBURG, W. A. J. (Ed.). Applications of model theory to algebra, analysis and probability. New York: Holt, Rinehart, and Winston, 1969. p. 109-122.

STROYAN, K. D.; LUXEMBURG, W. A. J. Introduction to the theory of infinitesimals. New York: Academic Press, 1976.

Artigo recebido em 04/12/2011. Aceito em 16/07/2012. 